\title{
A Novel Remote Control System for Air Conditioning in Low Carbon Emission Buildings Using Sensor Fusion and Mobile Communication Technologies
}

\author{
Wenjie Peng ${ }^{1}$, Daizhong Su ${ }^{2^{*}}$ and Martin Higginson ${ }^{3}$ \\ 1,2 Advanced Design and Manufacturing Engineering Centre, School of Architecture, Design and the Built \\ Environment, Nottingham Trent University, Nottingham, NG1 4FQ, UK \\ ${ }^{1}$ wenjie.peng@ntu.ac.uk ${ }^{2}$ daizhong.su@ntu.ac.uk ${ }^{3}$ martin.higginson@ntu.ac.uk \\ ${ }^{*}$ Corresponding author
}

\begin{abstract}
Water-cooled air conditioning units have huge potential for providing a low carbon emission solution to controlling the temperature and humidity of commercial and domestic buildings as they do not rely on hazardous refrigerant materials in their operation unlike most present devices. Currently, however, its use is limited in high humidity areas due to low cooling efficiency and no effective method for controlling this type of system remotely.

This research developed a novel remote-controlled system for use with air-conditioning units utilising solid desiccant dehumidifiers which can be employed to create low carbon emission buildings. The system employs multiple information technologies such as sensor fusion, digital input/output communication and mobile technologies to monitor and control the internal conditions of air-conditioned buildings and provides data collection, data processing, system control, and mobile access. The data collection module is built based on sensor fusion technologies to detect the operating status of the conditioned air. The real-time data processing module is implemented with feature extraction and non-linearization computing to process received data and transmit commands to a control unit. A case study involved monitoring and controlling the working conditions of a solid desiccant dehumidifier employed in an air conditioning unit. Remote control of the process was performed by tuning a NI-DAQ controller's parameters to regulate humidity, temperature, flow rates and other conditions in the unit. A mobile phone was utilised to demonstrate the remote-control function. The results prove that the system developed is successful in controlling the operation of water-cooled air conditioning units.
\end{abstract}

Keywords: Buildings; air condition; low carbon emission; solid desiccant dehumidifier; mobile phone; sensor fusion; control system; process control; web services; mobile network.

\section{Introduction}

These days, air conditioning is considered to be a crucial addition to most commercial buildings and its use in domestic environments is also becoming more widespread as residents seek to experience the maximum level of comfort available to support their modern lifestyles. From an environmental perspective, however, such systems consume large amounts of energy and contribute to pollution. Currently, most air conditioning devices operate using conventional refrigerants such as chlorofluorocarbons (CFCs) and hydrofluorocarbons (HFCs) [1], which generate considerable emission of greenhouse gases and other harmful materials, producing a negative environmental impact.

As a way of reducing environmental impact, water-cooled air conditioning systems have attracted attention, with such systems being cost-effective and not requiring potentially hazardous refrigerant pipes running through the house [5]. These systems operate based on water evaporative cooling, in which water absorbs heat from indoor air and transfers it outside to disperse in the atmosphere. Currently, water-cooled air conditioning systems for domestic use are quite rare, although some companies have made inroads into this market. For example, Olimpia Unico, an Italian company, have developed a chilled water air conditioning system for use in homes [5], whilst in the UK, P\&M Coppack Air Conditioning Ltd. have produced an Airwell water-cooled air conditioning system with independent water-cooled consoles which can be connected to the public water supply and are fitted with a modulating regulator valve to reduce water consumption [6]. LG on the other hand, provides a chilled water central air-conditioning solution which is ideal for industrial facilities, 
such as power plants and factories as well as district cooling [7]. With regards to control techniques for heating, ventilation and air conditioning (HVAC) systems, Abdul Afram et al. has conducted a review and considers that with recent advances in data storage, computing and communication devices, the implementation of an effective method to overcome the inherent issues in HVAC control should be possible [19].

A major problem of existing water based air conditioning units, is that the application of water evaporative cooling is often restricted in the UK and some areas of mainland Europe due to the relative higher humidity there. Theoretically, the evaporative cooling system is only suitable for hot and dry climates [15]. In moist conditions, air is not suitable for supply into buildings directly, as it achieves low cooling effectiveness and may cause distortion and rusting of susceptible materials [16]. In the UK and some European areas, the climate is humid all year around (e.g. relative humidity as high as 50\%-70\%) and doesn't change dramatically within the four seasons [17] [18], which therefore limits the application of water evaporative cooling somewhat.

Current water-based air conditioning systems focus on the cooling technique, but more attention could be paid to the problem of air humidity, which limits the capacity of evaporative cooling and affects the effectiveness of controlling the thermal environment. An effective solid desiccant dehumidification method is needed to address this problem.

The application of mobile phones to control heating, ventilation and air conditioning (HVAC) systems is currently limited to conventional refrigerant-based air condition systems [21]. The advantage of employing the mobile phone is that the user can regulate the building's temperature and humidity from a remote place to optimise energy efficiency and ensure comfortable conditions await on return. Ensuring smart devices such as mobile phones and sensing units [8] can be used in conjunction with water-cooled air conditioning systems is also desirable for the same reasons, but currently no application of their use in these systems exists [20].

Current climate change predictions indicate that the UK will have Mediterranean style weather conditions within the next 50 years, with heat waves already becoming more prevalent [2]. Present regulations are pushing towards Zero Carbon homes by 2050, when it is possible that traditional high-energy equipment will be required to provide cooling in both commercial buildings and domestic dwellings of older construction, creating higher demands of energy $[3,4]$. This research contributes to solving these issues by providing a lowenergy solution for the cooling of older buildings.

To meet the challenges stated, the research presented in this paper discusses the development of a novel conditioning system using water as a coolant and a solid desiccant for dehumidification. The water, which is environmentally friendly, replaces more harmful refrigerants used to operate conventional air-conditioning systems in buildings. The dehumidification method adopted overcomes the problems of current water based conditioning systems to ensure appropriate levels of control of the building's environment, which is a novel application [13].

Importantly, the system described is CFCs and HFCs free and can be driven by a natural energy source, such as the evaporation of water. The solid desiccant dehumidification technology, which is developed in this project, will be important for advancing environmentally friendly solutions for achieving comfortable working and dwelling conditions in buildings. Since the amount of electricity required to drive the system is low, it will be suitable for adaption using renewable energy sources, such as solar and wind energy. The solid desiccant dehumidification technology has not been investigated thoroughly so far and hence greater research is necessary in this area [5].

As stated, the use of mobile phone and sensing technologies have great benefit when applied to air conditioning systems [12] but to date they have not been applied in water-cooled systems [10]. To address this, mobile communication technology is applied, which allows the user to remotely control the functions of air conditioning (e.g. the control switch of the dehumidification system) via mobile phone, in order to set optimum humidity and temperature from distance. Furthermore, mobile phone technologies are combined with sensing technology to develop a new means for real-time monitoring and controlling the working conditions of the air-conditioning units, which ensures the operation of the solid desiccant dehumidification system, which is a novelty in this research.

In the following sections, mobile communication and control of the system is reported, including the use of multi-sensor application, digital I/O methods, I/O control (hardware), real-time condition data processing and communication through a mobile phone network. Other aspects related to this research will not be addressed due to the word count constraints.

\section{System Overview}


Figure 1 shows the water-cooled air condition system in low carbon emission buildings, which applies a new type of solid desiccant dehumidifier to ensure suitable control of temperature and humidity.

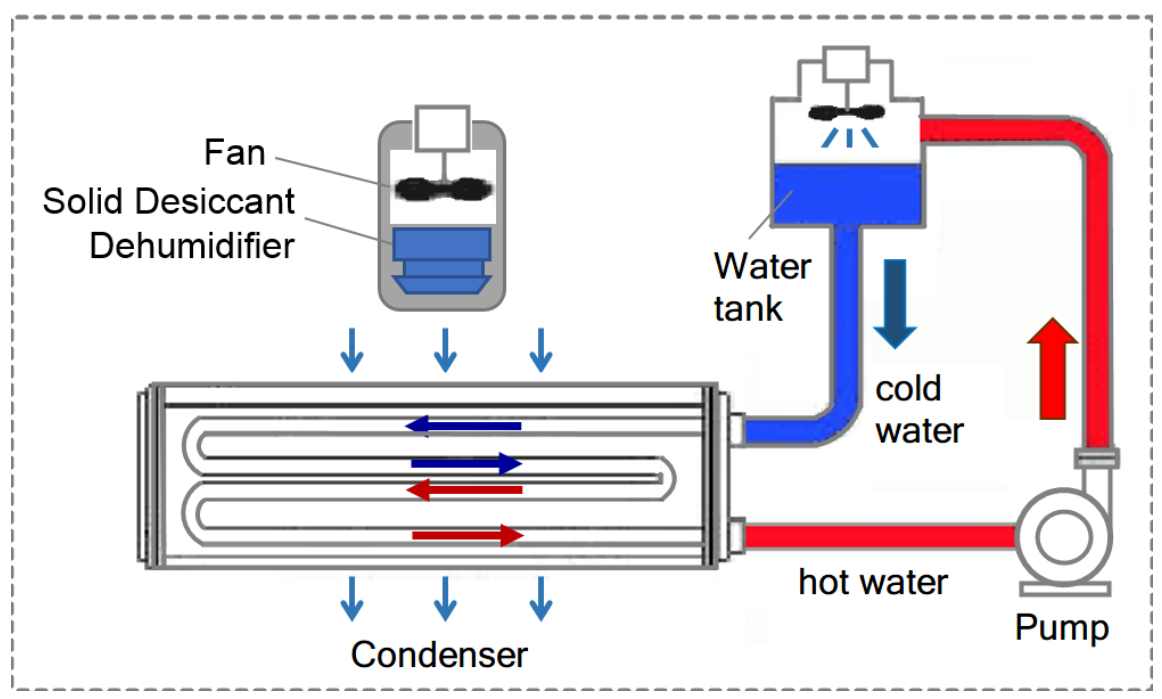

Figure 1: Water-cooled air condition with solid desiccant dehumidifier

A novel system for monitoring the working conditions of water-cooled air condition units in low emission buildings has been developed. The system regulates the humidity within the room via monitoring the flowrate, moisture and temperature of the air that flows through the air-conditioning unit, in order to satisfy the requirements for water evaporating cooling. Importantly, the sensing technologies, such as sensor fusion and digital I/O with control output are applied, which solve the problem of data fusion of multiple complex working conditions. Sensor fusion combines multiple sensors, which are not specified to originate from identical sensors, to capture physical quantities of different working conditions of air-conditioning. Digital I/O (input/output) is used to achieve dual communication with input and output devices, such as retrieving the working condition data from the sensors and transferring the command to the control unit.

The system consists of a solid desiccant dehumidifier for reducing air moisture, an air conditioning unit, a fan with adjustable speed, a data acquisition device for digital I/O (input/output) control, sensors for data acquisition for multiple working conditions, a web camera, a host computer and mobile facilities, which are all shown in Figure 2.

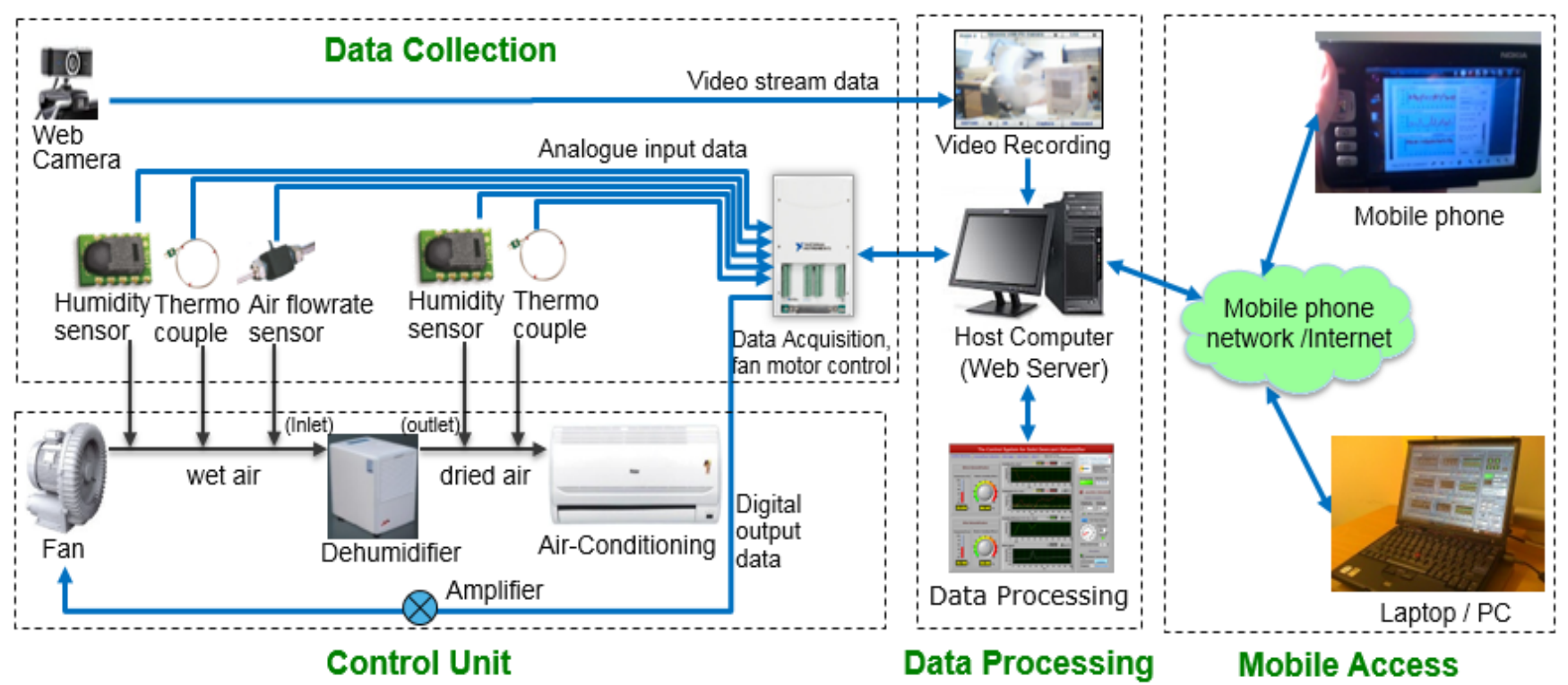

Figure 2: Mobile control system for air-conditioning with solid desiccant dehumidifier

Within this system, the solid desiccant dehumidifier plays a significant role in tuning the humidity of air. The air dehumidification is performed using the solid desiccant within the dehumidifier. The dehumidifier includes an inlet and an outlet for air-flow. When the fan blows the moist air into the inlet of the dehumidifier 
at a certain flow-rate, the solid desiccant conditions the flowing air by reducing the moisture The lowhumidity air is then outputted via the outlet of the dehumidifier and subsequently conveyed to the airconditioning unit for water evaporative cooling. Through this method, the humidity of the room is reduced.

In order to track the changes in the working conditions of the air (i.e. before and after conditioning it), a fusion of sensors and relevant measurement devices are utilised for data collection, and an internet-enabled host computer employed for data processing and real-time display. The humidity of the conditioned air at the outlet is tuned via the control of the fan's speed, utilising a data acquisition device (DAQ) with digital output control and a current amplifier device. Importantly, users are able to control the humidity in the room remotely by mobile device, which is an innovation.

To achieve the above goal, the system provides the following four functions: data collection, data processing, system control and mobile access, which are detailed as follows:

(1) Data collection is implemented with the sensory technique based on the digital I/O interfaces to collect the data of working conditions from the solid desiccant dehumidifier. In order to monitor the operational status of the dehumidifier, the sensors are placed at both the air-inlet and air-outlet of the dehumidifier to acquire working conditions data of the air, including humidity, temperature and flow rate. To do so, a humidity sensor, a thermocouple and an air-flow rate sensor are installed at the air-inlet of the dehumidifier and a humidity sensor and a thermocouple located at the air-outlet of the dehumidifier, as shown in Figure 2.

The data acquired by the above-mentioned sensors are transmitted to the host computer via the data acquisition device (DAQ) for further processing. In the meantime, a web camera is used for transferring the on-site video to the host computer. For more details of the acquisition and calibration of the working condition data of the solid desiccant dehumidifier, please see section 3.1 Data Collection.

(2) Data processing is applied to handle all the data and commands related to monitoring, control and mobile access. The host computer operates as the central console to acquire the working condition data of the solid desiccant dehumidifier, and if necessary, transmit the commands to the control unit, such as the fan's motor. By controlling the fan's speed, the humidity of the room is adjusted. Also, the host computer provides a web-based software interface (i.e. web service) for connection with mobile devices, such as mobile phones, tablets and laptops. Therefore, the mobile user can connect to the computer remotely and is allowed to send the commands to control the fan. The related software is developed using LabVIEW programming software. For more details of the data processing technique applied in the host computer, please see section 3.2 Data Processing.

(3) The System control is implemented to control the operation of the dehumidifier and fan which provides the source of air-flow for the solid desiccant dehumidifier. The rate of the air-flow within the dehumidifier is determined by the speed of the fan. The faster the fan rotates, the greater the air flows through the dehumidifier within a given time. When the amount of air-flow through the dehumidifier per second exceeds the amount that the dehumidifier can deal with, i.e. the upper limit of the capacity of air-flow dehumidified by the solid desiccant, the control unit decreases the fan speed immediately in order to slow down the air's flow-rate. To achieve this, the control unit adopts the DAQ device with digital control to an analogue current amplifier, in order to regulate the operation of the fan. The adjustment of the fan's speed can be performed by tuning the output voltage values on the DAQ device and amplifying the output current values on the current amplifier device. For more details of the control method with regards to the DAQ device, please see section 3.3 System Control.

(4) Mobile access enables the user to view the working condition data with their smart device and adjust the air humidity within the rooms as required via a mobile phone network. The necessary web-based application software has been developed by the following software developing tools:

- LabVIEW Developer Suite, a G-language based graphical programming environment, to develop the server-end applications for data acquisition and decision making (system control);

- Adobe Flex Builder, a programming development environment, to develop the graphic user interfaces (GUI) and script programmes for remote communication of the client end (user side) with the server end;

- Adobe Flex Media Server (FMS), to develop the media-driven code for transferring the video stream data from the host computer to mobile phones at the client end. For more details of the programming of remote communication between the host computer and the mobile phones, please see the section 3.4 Mobile Access. 


\section{Technologies Utilised in the Air Conditioning System}

The system employs sensor fusion, data processing with feature extraction, system control and mobile communication technologies in order to monitor and control the working conditions of the solid desiccant dehumidifier and maintain the normal operating status of the air-conditioning unit.

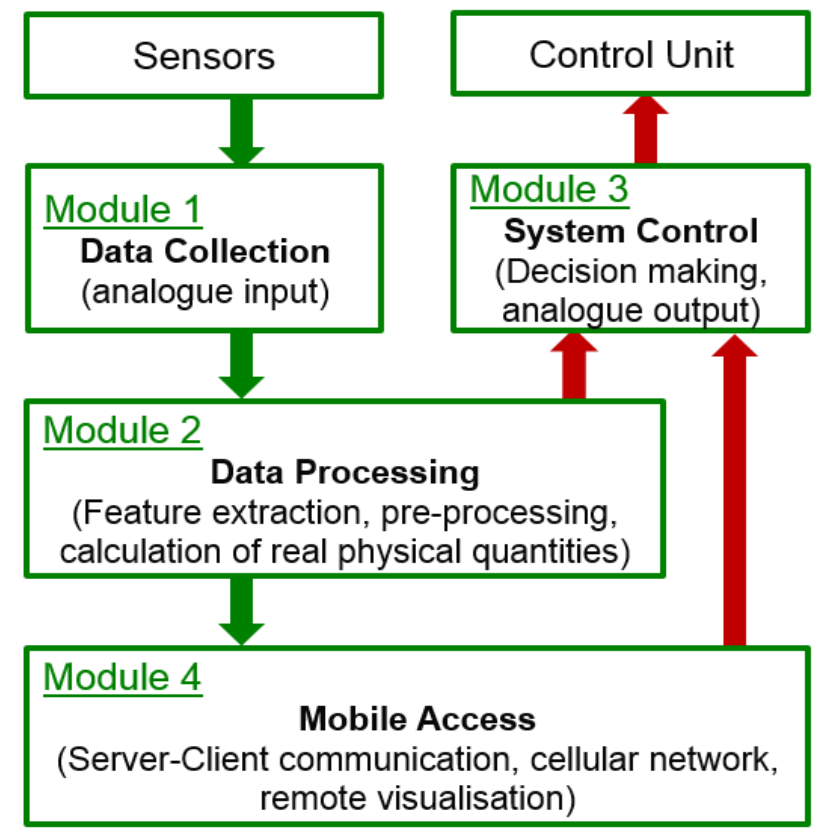

Figure 3: The flow of monitoring and control of the dehumidifier system

Figure 3 shows the workflow of the system and the functionality of each module within it. First, the data collection module collects working condition data from the dehumidifier via a series of sensors. The captured data are then processed to actual physical quantities (humidity, temperature, and air flow-rate) within the data processing module via applying feature recognition, pre-processing and non-linearization calculations. Subsequently, the system control is implemented to identify the overload / failure condition of the dehumidifier and make decisions based on the actual physical condition data. When detecting a failure, the control commands are immediately transmitted to the control unit (e.g. fan motor) for regulating the working conditions of the dehumidifier (e.g. air humidity). A significant feature of this system is that users are permitted to view the working condition data on their mobile phones, as well as control the working condition by submitting commands to the control unit online.

The technologies applied in the remote control system are detailed in the following sub-sections.

\subsection{Data Collection}

Data collection is conducted using multiple sensors and the data acquisition device (DAQ) based on the data input/output $(\mathrm{I} / \mathrm{O})$ communication interface. The sensors are employed to collect the working condition data of the solid desiccant dehumidifier, such as humidity, temperature and air flow rate, which include the following:

(1) Two humidity sensors to acquire data at the inlet and outlet points of the solid desiccant dehumidifier respectively.

(2) Two thermocouples (temperature sensors) associated with a signal conditioner, to acquire the temperature data at the inlet and outlet points of the solid desiccant dehumidifier respectively. The signal conditioner provides the temperature compensation for the cold-junction of the thermocouples, in order to ensure the accuracy of the temperature data measured. Also, it is applied for amplification and filtering of the low-voltage signals generated by the thermocouples. The application of the signal conditioner reduces the measurement error of the temperature data; the measurement error is less than $0.1 \%$ at $0-70{ }^{\circ} \mathrm{C}$. 
(3) A flow rate sensor, for measuring the air flow rate into the solid desiccant dehumidifier. The sensor enables a quick response to monitor the humid atmosphere and has a high accuracy with less than $2 \%$ measurement error.

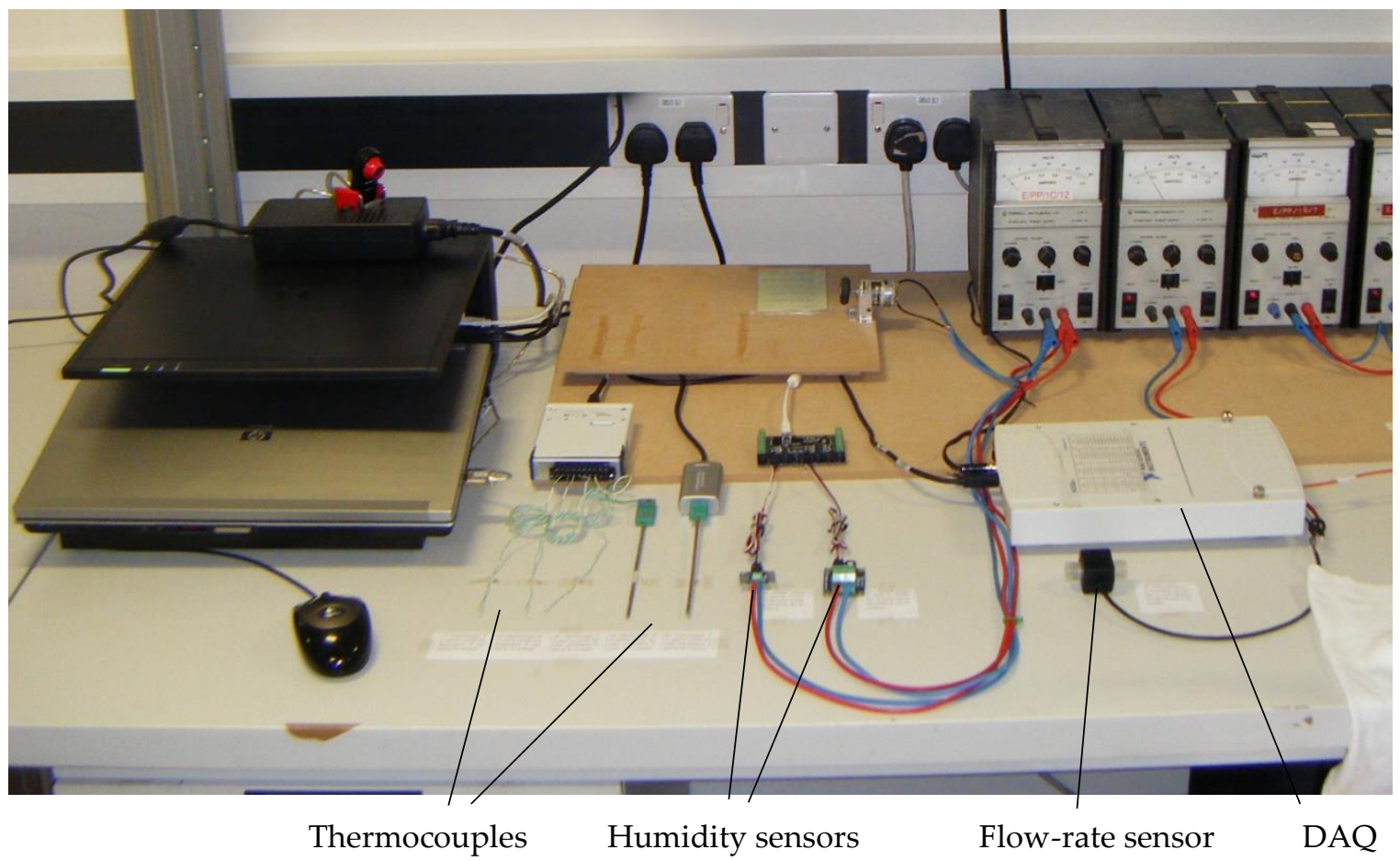

Figure 4: Data collection module, including humidity sensors, thermocouples, air flow-rate sensor and associated data acquisition device (DAQ)

Figure 4 shows the thermocouples with K-type miniature plugs/probes together with the high precision humidity and flow-rate sensors which are used for the measurement of working condition data. The sensors transmit the working conditions data of the solid desiccant dehumidifier to the DAQ in the form of an analogue input voltage. The DAQ then converts the analogue input voltage data to the digital input data, which can be read by the host computer.

The DAQ contains the data input/output (I/O) communication interfaces, for example, the analogue input (AI) interface and the digital counter (DC) interface, in order to transmit the digital input data with the physical information of the working conditions to the host computer for further processing. The analogue input (AI) interface is used to collect the analogue input voltage data based on the differential calculation method. The sampling rate of the analogue input interface is $10 \mathrm{kHz}$ and its data length is 100 per channel. The analogue input voltage values range from 0 to 10 volts [9]. The digital counter (DC) interface adopts the pulse counting method to collect data from a sensor. It has the capability of high-rate computation of data; the sampling rate of the digital counter is $80 \mathrm{kHz}$ [9]. In this research, the analogue input interface is used, which transmits the digital input data of humidity, temperature and flow-rate to the host computer.

Figure 5 shows the graphic user interface and the man-machine interactive interface, which display the working condition data of the air-conditioning system in real time. First, the working condition data are captured from the sensors at a rate of $10 \mathrm{~K}$ per second and then they are transmitted via the DAQ device to the host computer for further processing, such as feature extraction. 


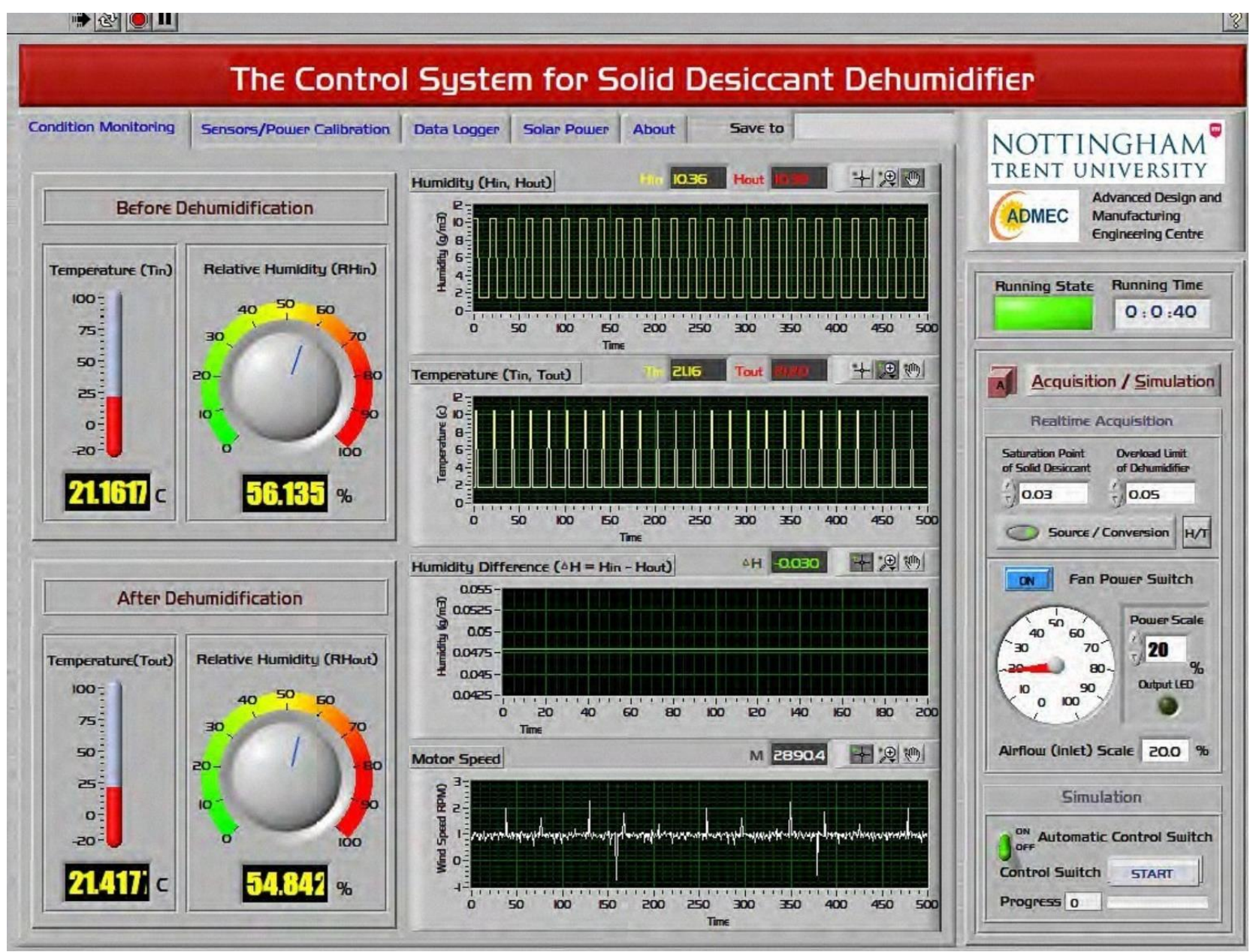

Figure 5: Graphic user interface for the display of the working condition data

\subsection{Data Processing}

The data processing module is utilised to process the digital input data, which are obtained by the host computer, into the actual physical quantities of working conditions. It provides the functions of feature extraction, pre-processing, calculation of real physical quantities and display of the data/results on the monitor, which are detailed as follows:

(1) Feature extraction

The data that are captured from the humidity, temperature and flow-rate sensors are shown in the form of electronic impulse, which are composed of four types of feature parameters: RMS, valley value, peak value and period [8]. The peak value reflects the analogue voltage value of humidity/temperature condition which is to be extracted. The feature extraction software has been developed for extracting the impulse's feature parameters as shown in Figure 6. Based on this software, the impulse's peak values of humidity and temperature are obtained as well as the impulse frequency of the air flow.

(2) Pre-processing

In order to ensure the accuracy of the humidity and temperature measurements, the data are processed using the following procedure:

a) The data are categorised into ten groups and each group has 1024 data.

b) The data of each group are calculated to obtain a root-mean-square (RMS) value of the group.

c) The average of the RMS values of ten groups is used as the value of working condition data at a time point.

With the above method, the abnormal data (also called invalid data) are removed, which ensures the calculation error is lower than one percent. 


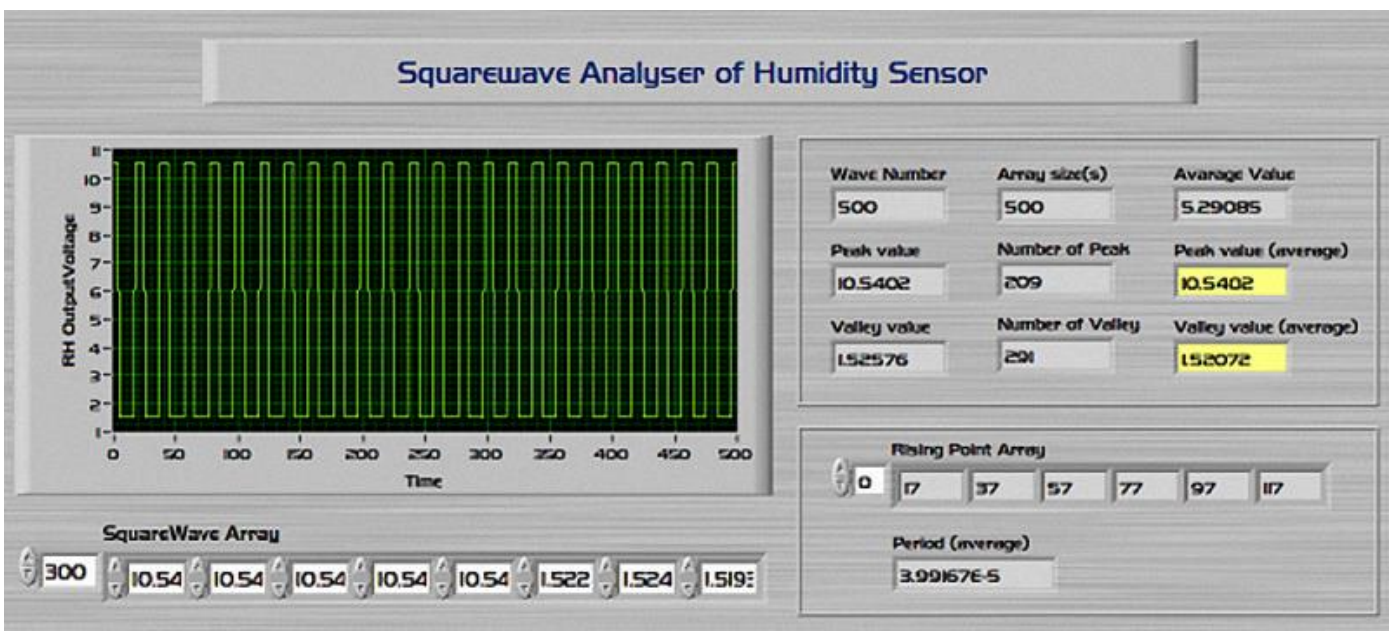

Figure 6 (a) : Feature extraction of the humidity sensor

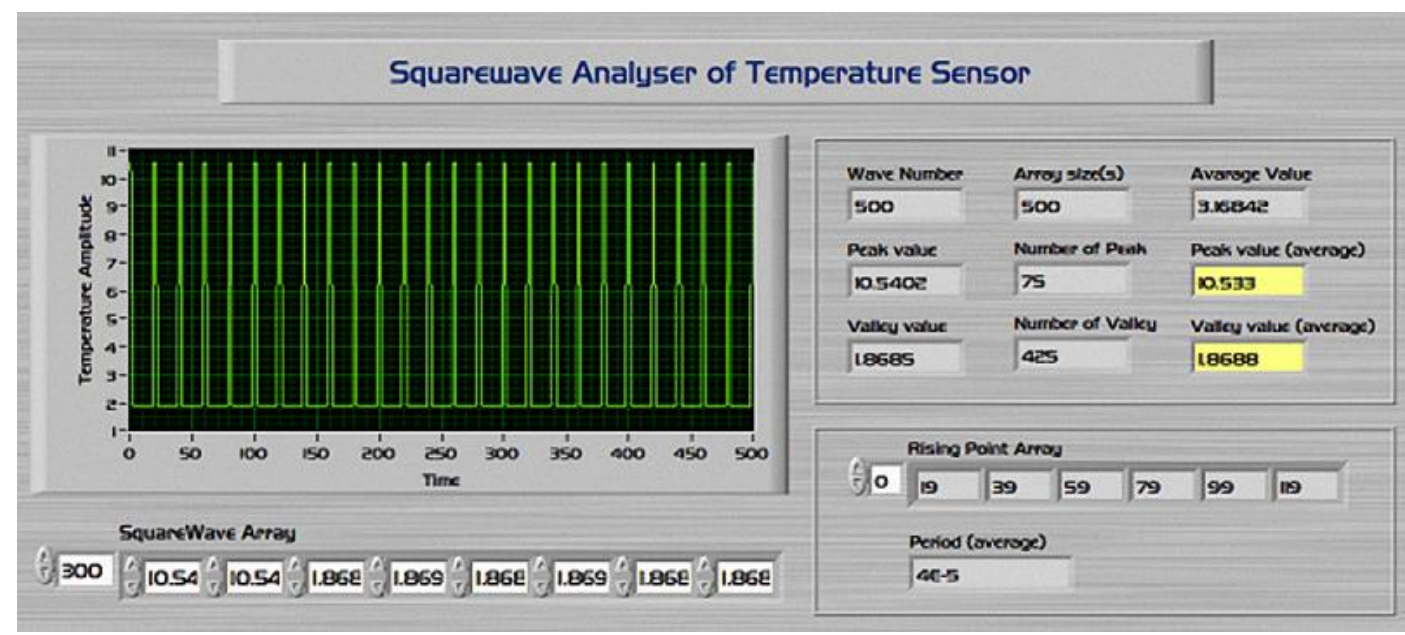

Figure $6(b)$ : Feature extraction of the temperature sensor

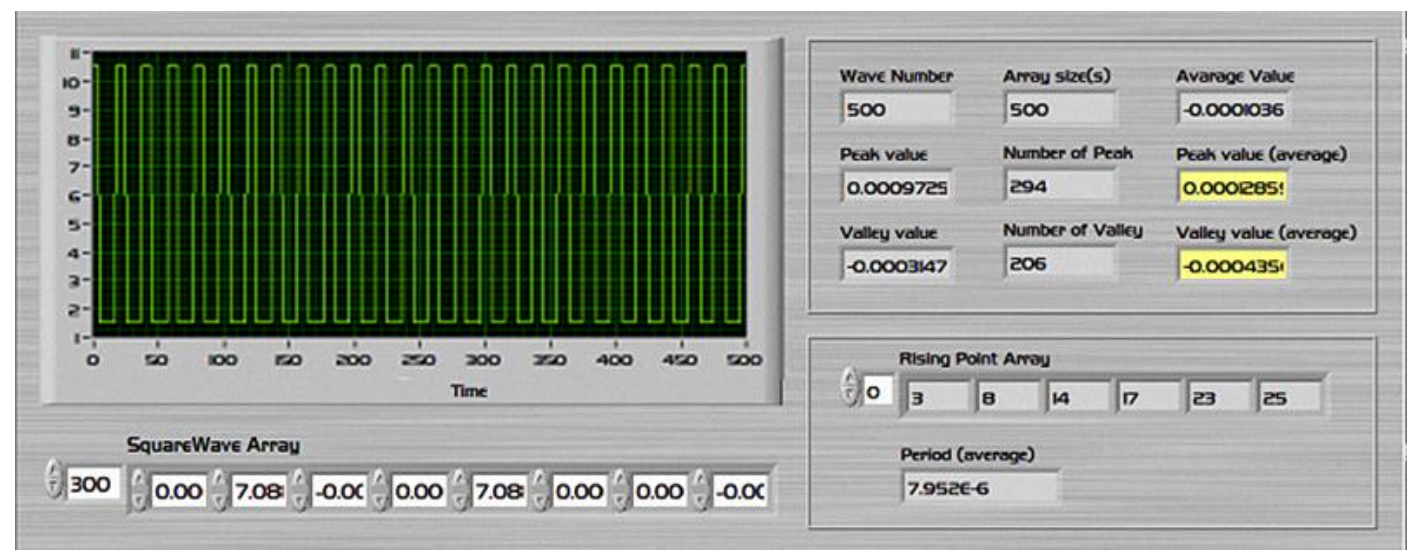

Figure 6 (c) : Feature extraction of the flow-rate sensor

(3) Calculation of physical quantities

The digital input data are converted to real physical quantities which can be understood by the user. Usually, the data captured are not linearly proportional with the real physical quantities and therefore a nonlinearization based conversion is applied. The following formulas are used for calculating the real physical quantities, including humidity (i.e. both relative humidity and absolute humidity), temperature and flow-rate: 
Relative Humidity:

RH $(\%)=(h \times 0.1906)-40.2$

Temperature:

$\mathrm{T}\left({ }^{\circ} \mathrm{C}\right)=(\mathrm{t} \times 0.22222)-61.11$

Absolute Humidity: $\quad \mathrm{H}\left(\mathrm{g} / \mathrm{m}^{3}\right)=216.7 \times \frac{R H}{100} \times 6.112 \times \frac{e^{\left(\frac{17.62 \times T}{243.12+T}\right)}}{(273.15+T)}$

Flow Rate:

FR (litre/hour) $=f * 60 / 7.5$

Where the variable ' $h$ ' refers to the data obtained from the humidity sensor; ' $t$ ' represents the data obtained from the temperature sensor (thermocouple); and ' $f$ ' represents the impulse frequency data of the flow-rate sensor.

\subsection{System Control}

Within the working condition information obtained, the humidity data is the key indicator which is used to identify if the dehumidifier is overloaded. Overloading of the dehumidifier arises from excessive air flow, which is not able to be conditioned by the solid desiccant in the dehumidifier and hence could result in a failure of air dehumidification.

The identification of dehumidifier overload is found by calculating the change in humidity of the air within the dehumidifier, which is indicated as $\Delta \mathrm{H}$. When the dehumidifier is idle or not operational due to the failure, $\Delta \mathrm{H}$ is zero. With the dehumidifier functioning, $\Delta \mathrm{H}$ increases gradually and therefore $\Delta H$ can be used to judge the operating status of the dehumidifier.

In this research, the humidity of the air-inlet and air-outlet of the dehumidifier are indicated as $\mathrm{H}_{\text {in }}$ and Hout respectively. The change of air humidity can be expressed by the following equation:

$$
\Delta \mathrm{H}=\mathrm{H}_{\text {in }}-\mathrm{H}_{\text {out }} \quad\left(\text { unit: } \mathrm{g} / \mathrm{m}^{3}\right)
$$

Here, $\Delta \mathrm{H}, \mathrm{H}_{\text {in }}$ and $\mathrm{H}_{\text {out }}$ refer to the absolute humidity quantities. Correspondingly, $\Delta \mathrm{RH}, \mathrm{RH}$ in and $\mathrm{RH}$ out represent relative humidity quantities. The air humidity ratio of the dehumidifier is also an important indicator, which is calculated as follows:

$$
\Delta \mathrm{H}_{\mathrm{c}}=\left(\mathrm{H}_{\text {in }}-\mathrm{H}_{\text {out }}\right) / \mathrm{H}_{\text {in }}=\Delta \mathrm{H} / \mathrm{H}_{\text {in }}
$$

where $\Delta \mathrm{H}_{\mathrm{c}}$ is within the range 0 to 1 .

$\Delta \mathrm{H}$ is related to the rate of air flow through the dehumidifier (the air's flow-rate represents the amount of air flow passing through the dehumidifier within a given time). If the air flows fast, then $\Delta H$ drops. When the air flow-rate arrives at a certain value, $\Delta \mathrm{H}$ will in turn reduce significantly (e.g. approaching zero), which means that the amount of air-flow through the dehumidifier is exceeding the value of the upper limit that the dehumidifier can deal with.

It is important therefore to set the value of the lower limit of $\Delta \mathrm{H}$. When $\Delta \mathrm{H}$ is less than the value specified, the system will identify that the dehumidifier is overloaded. In addition to $\Delta \mathrm{H}$, the temperature value may assist in judging the operating status of the dehumidifier. In general, temperature increases with the drop of air humidity.

Once the dehumidifier overload is identified, the system is able to make a response immediately, which is implemented as follows:

1) Launching the pre-set processing procedure/programme by transmitting a digital control command of the decelerating fan's speed to the DAQ card, e.g. decelerating by $5 \%$ of the fan's rotating speed.

2) Based on the digital-to-analogue $(\mathrm{D} / \mathrm{A})$ conversion function, the $D A Q$ device converts the digital data of the control commands to the analogue output voltage, which is then transmitted to the fan motor. In this research, the DAQ works not only as a data collection device but also a machine controller. It has a two-way communication capability for input and output, such as analogue input for data acquisition and analogue output for control [14]. When the analogue output is triggered, the DAQ 
converts the digital command data into an analogue output voltage, which is then transferred to the unit controlled.

3) The analogue output voltage controls the fan motor speed, slowing it down as required. Further, if the air flow-rate through the dehumidifier is reduced, an increase in $\Delta \mathrm{H}$ results.

4) If $\Delta \mathrm{H}$ is less than the value of the lower limit, then the steps from 1) above can be repeated until $\Delta \mathrm{H}$ reaches the allowable working range.

If $\Delta \mathrm{H}$ cannot be adjusted to be in the safe-working range required by the dehumidifier using the above procedure, the system is to power off the fan and terminate the dehumidifier operation. In the meantime, the system will display warning messages on the monitoring panel to inform on-site operators as well as generating an analytical report. The course of action highlighted above ensures that the dehumidifier is not damaged, which effectively solves the dehumidifier's overloading problem.

\subsection{Mobile Access}

This module utilises the server-client networking structure, which facilitates the mobile communication between the server side (host computer) and the client side (mobile phone), as shown in Figure 7. The application programming interface (API) for the connection is the REST-based web service (Representational State Transfer), which is responsible for receiving the request from the mobile phone at the client side and then to interact with the three modules (data collection, data processing, and system control) and the web camera for on-site visualisation.

When the mobile user sends a request with the control commands to the server via the cellular phone network, the web service transmits the request to the data collection / processing module for further processing. Based on the request received, the control commands will be transmitted to the control unit (e.g. fan motor) to perform the required tasks.

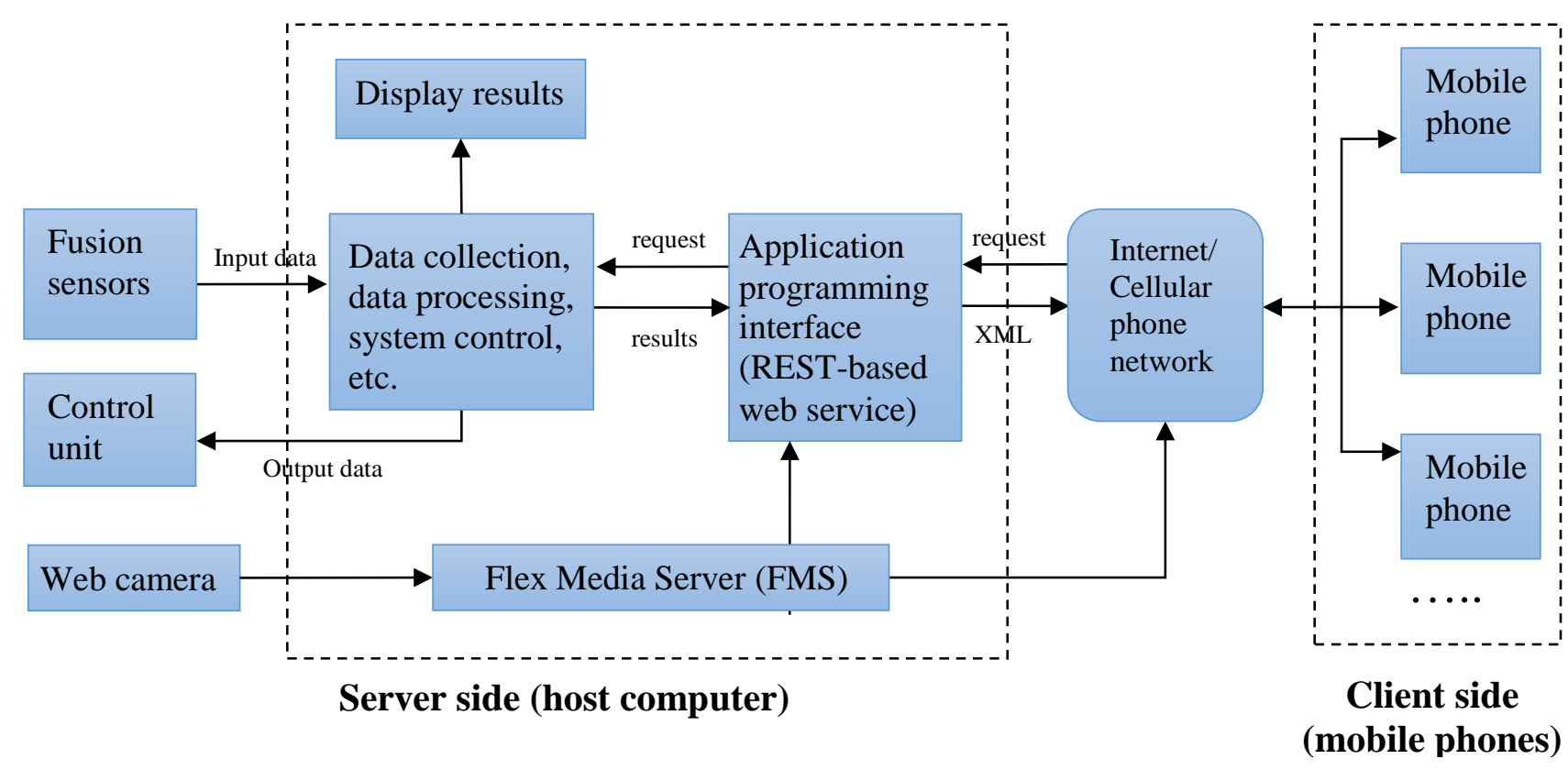

Figure 7: Overview of the communication between the server and the client using the internet and the cellular phone network

In response to the client-end user, the working condition data of the dehumidifier is sent to the mobile phone client in the form of XML (Extensible Mark-up Language) over the internet/cellular phone network. $\mathrm{XML}$ is an efficient and reliable data-carrier, used in the web service and conveyed over the internet and allows data to be converted into a standard form and transferred to the client-end user. Figure 7 shows the remote communication between the server and client side via the internet and cellular phone network. The development of the server-client network is detailed in the following sub-sections.

(1) Development of Server Side 
The server side consists of a host computer, a monitor panel, a web camera and a network interface for communication with the client user. The web camera is used to capture the video stream for remotely monitoring the site.

The host computer provides the functions of real-time data acquisition, data processing and system control along with facility to communicate with the client-end mobile phones. The above functions are programmed by the LabVIEW Developer Suite development software.

The server programme contains several sub-programmes, which are called blocks in LabVIEW. Each block performs an independent function, e.g. data acquisition, analogue-to-digital or digital-to-analogue conversion and data transfer over the network. These blocks utilise the common computer/networking resources, such as network ports, transmission protocols and application programming interfaces, and transfer the data/information to each other.

The inter-operability of programme blocks is performed by the network-based shared variables (GSV), which are used to transfer different types of data, such as two-dimensional waveforms graphs and tables. As an important interactive function used in LabVIEW, GSV can effectively transmit data/information to the interface specified in the server, i.e. the application programming interface (API), for communication with the client.

As a standard application programming interface, the REST-based web service (Representational State Transfer), is applied to create a remote connection between the host computer on the server side and mobile phone on the client side. The web service enables the server to transmit the working condition data to the user's mobile device and hence the user is able to retrieve the data over the internet when required. When the user tries to control hardware devices, such as adjusting the fan's speed, the web service can provide the realtime online connection, allowing for the user to access the server to transmit the control commands to the devices remotely. The web service secures the communication between the server and the client and ensures the compatibility of different computer languages/platforms.

The implementation of the REST-based web service starts by processing the data to the XML form. Using the following XML syntax, the data/information, such as working conditions data captured by humidity and temperature sensors, real physical quantities and system message and parameters, can be encoded in the XML as follows:

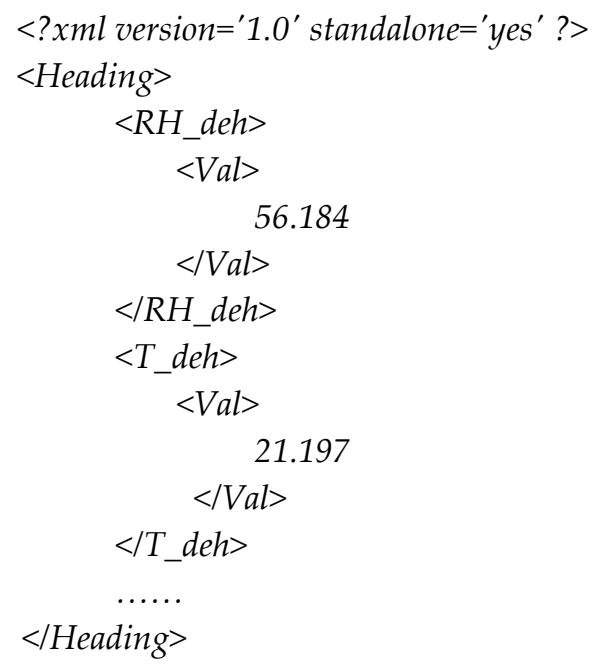

Within the above XML code, '56.184' and '21.197' refer to relative humidity data and temperature data after air dehumidification, respectively. The tags $<\mathrm{RH} \_$deh $>$and $</ \mathrm{RH} \_d e h>$ are used to define a record with '56.184', and the tags $<$ T_deh $>$ and $</$ T_deh $>$ use the same method to define a record for '21.197'. The tags $<$ Heading $>$ and $<$ /Heading $>$ specify a heading of the XML code including all the records [11].

Using the above syntax, the data/information is encoded to the XML, which is then transmitted as a response to a data buffer in the memory using the Response function of LabVIEW. When the server receives a user request from the client, the response, i.e. the XML code, which remains in the data buffer, will be sent to the client.

The real-time video stream for monitoring the operation of the dehumidifier is also sent to the user together with the working condition data. The video stream is acquired by the Web camera and then processed 
by Flex Media Server (FMS) software, which encodes the captured video to stream data and broadcast it to the client side. The process of publishing and transferring the stream data to the user is programmed based on the MXML language used in Adobe Flex builder.

\section{(2) Development of the Client Side}

The client end of the system allows several mobile phones to access the server to acquire the working condition data concurrently, including graphs, tables and video via the cellular phone network or the internet. It also allows an authorised user, such as a technician, to log into the server-side host computer to control the operation of the dehumidifier i.e. by delivering requests with control commands to the host computer. Such a control method allows the engineers/technicians to test the functionality and reliability of the system.

The client software (i.e. mobile phone app), including graphic user interfaces and script, is developed using Adobe Flex Builder and Open-plug programming environments. The graphic user interfaces are applied to show the data of the working conditions of the dehumidifier, such as humidity $\left(\Delta \mathrm{H}, \mathrm{H}_{\mathrm{in}}, \mathrm{H}_{\text {out }}, \Delta \mathrm{RH}, \mathrm{RH} \mathrm{H}_{\mathrm{in}}\right.$ $R H_{\text {out }}$ ), temperature (T) and flow-rate (FR). The graphic user interfaces also allow the user to configure the information required for access to the server and enter the parameters related to the control devices.

The script associated with the graphic user interfaces is developed to deliver the data/results received from the host computer to the graphic user interface for the mobile phone display and in addition, to send back the user requests such as the system parameters and control commands.

The communication with the host computer is implemented based on the REST-based web service, which is the same as the application programming interface used on the server side. The mobile phone can send requests with commands/parameters to the host computer via the web service, whilst it can also retrieve responses from the host computer in XML form and address the responses to display the data/results on the graphic user interface.

\section{Case Study}

The developed system has been successfully applied to control and monitor the working conditions of the solid desiccant dehumidifier used in an experimental air conditioning environment. The data acquisition hardware contains a NI-DAQ6259 data acquisition device, two humidity sensors, two thermocouples (temperature sensors) and a flow-rate sensor. The sensors are installed at the air-inlet and air-outlet locations of the dehumidifier respectively in order to monitor the humidity, temperature and flow rate at those positions. The DAQ sampling rate is $10 \mathrm{kHz}$ and its data length is 2048; the analogue input/output voltage ranges from 0 to 10 volts. The speed of the fan can be measured by a read-out of the operating voltage and the maximum no-load speed. In the set up shown below (and detailed in Figure 12), a Nokia phone is used to demonstrate the control functions of the system.

The work flow of the solid desiccant dehumidifier can be divided into three phases: start-up and steady operation of the dehumidifier, an overloading (failure) test and mobile control. The experiments conducted relative to the three stages are detailed in the following sub-sections.

\subsection{Start-up and operation of the dehumidifier}

Figure 8 shows the real-time monitoring of the dehumidifier system. The working condition data were acquired from the dehumidifier and displayed in the graphic user interfaces in a real time. Initially, $\Delta \mathrm{H}$ (change of air humidity of the dehumidifier) was $0 \mathrm{~g} / \mathrm{m}^{3}$, as shown in Figure 9 (a).

The operation of the dehumidifier and fan starts by sending the control commands to the DAQ device. When the DAQ device was triggered, the dehumidifier and the fan were launched, followed by a gradual increase in $\Delta \mathrm{H}$. The DAQ device applies two analogue output channels, Channel- 1 and Channel-2, to control the operations of the dehumidifier and the fan, with the following functions:

(1) Channel-1 supplies $10 \mathrm{~V} / 0 \mathrm{~V}$ to the dehumidifier to control the on / off function. When $10 \mathrm{~V}$ is outputted it starts operating and when $0 \mathrm{~V}$, it stops.

(2) Channel-2 supplies $0 \mathrm{~V}-5 \mathrm{~V}$ to the fan to control its speed, which is adjustable over the range from 0 to 4500RPM. The output voltage is proportional to the fan's speed. A voltage meter was applied to measure the voltage applied to the fan. 
Figure 9 (a) and 9 (b) shows the changes of $\Delta \mathrm{H}$ and the fan's speed respectively. It is clearly observed that at the time of 30 seconds, $\Delta \mathrm{H}$ increased to approximately $8.5 \mathrm{~g} / \mathrm{m}^{3}$, which reaches the maximum value of $\Delta \mathrm{H}$ that the dehumidifier can work to. This corresponds to a fan speed of around 2400RPM. The voltage supplied to the dehumidifier and fan was measured to be $10 \mathrm{~V}$ and $2.7 \mathrm{~V}$ respectively, using the voltage meter. This indicates that the dehumidifier works normally during this period to generate low-humidity air.

Increasing the fan's speed by tuning the DAQ device's control parameters created a greater air flow-rate through the dehumidifier. $\Delta \mathrm{H}$, however, remained less than $9 \mathrm{~g} / \mathrm{m}^{3}$ under normal operating conditions.

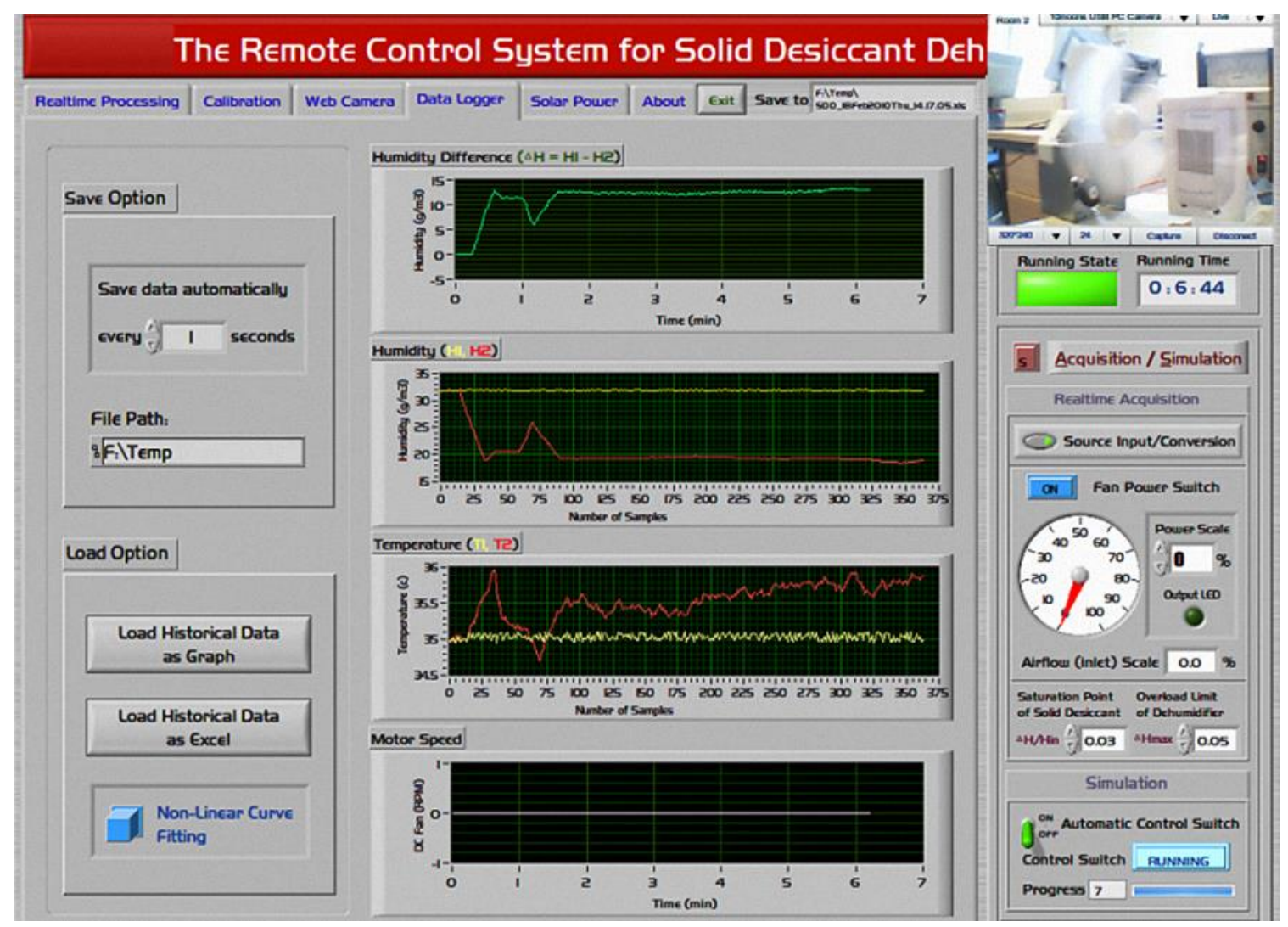

Figure 8: Real-time monitoring, control, and video visualisation of solid desiccant dehumidifier

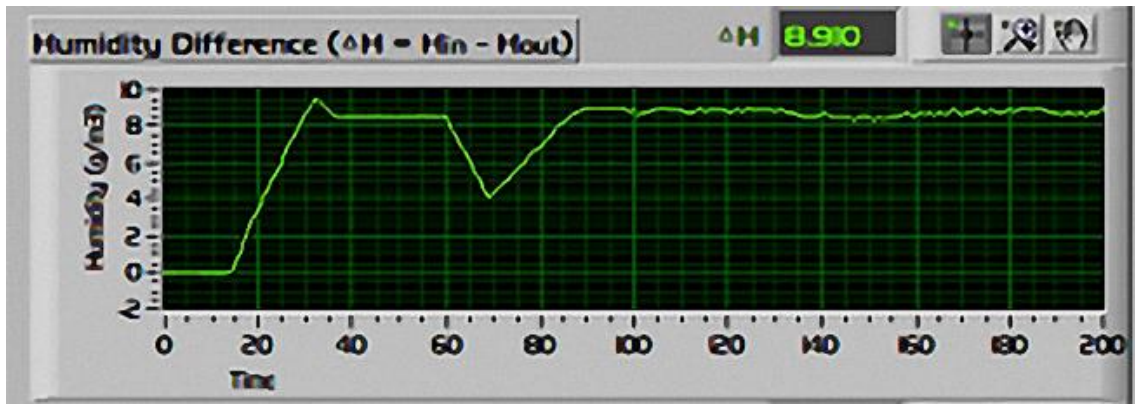

Figure 9 (a) : Tracking AH change over time

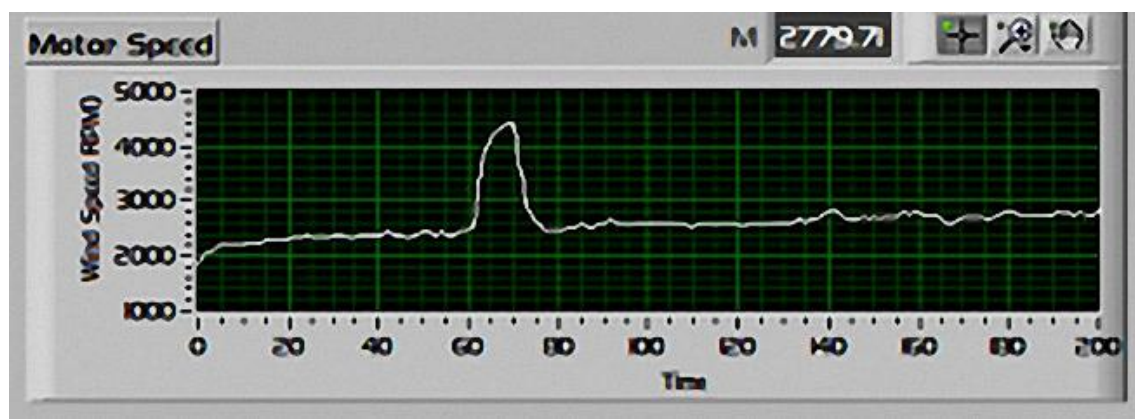

Figure $9(\mathrm{~b})$ : Tracking fan speed over time 


\subsection{Overloading/failure testing of the dehumidifier}

When the air flow was extremely fast, $\Delta \mathrm{H}$ dropped rapidly which indicates that the dehumidifier was overloaded. At a time of 70 seconds, the fan speed went up to $4500 \mathrm{RPM}$, while $\Delta \mathrm{H}$ fell to $4 \mathrm{~g} / \mathrm{m}^{3}$. The control system soon detected this status via the value of $\Delta \mathrm{H}$. Under the automated control mechanism of the system, the command to decelerate fan speed was transmitted to the DAQ device, which then outputted the analogue output voltage to the fan motor and regulated the fan's speed to slow down air flow-rate in the dehumidifier. At the time of 93 seconds, the fan's speed had dropped to 2500RPM and $\Delta \mathrm{H}$ rose to $8.5 \mathrm{~g} / \mathrm{m}^{3}$. Under these conditions, the dehumidifier returned to normal operation, as shown in Table 1.

The operating process of the dehumidifier is summarised in the following table:

Table 1: Working conditions of the solid desiccant dehumidifier

\begin{tabular}{|l|c|c|c|c|c|c|c|}
\hline Stage & $\begin{array}{c}\text { Time } \\
(\mathrm{Sec})\end{array}$ & $\begin{array}{c}\mathrm{RH} \text { in } \\
\left(\mathrm{g} / \mathrm{m}^{3}\right)\end{array}$ & $\begin{array}{c}\text { RHout } \\
\left(\mathrm{g} / \mathrm{m}^{3}\right)\end{array}$ & $\begin{array}{c}\Delta \mathrm{H} \\
\left(\mathrm{g} / \mathrm{m}^{3}\right)\end{array}$ & $\Delta \mathrm{H}_{\mathrm{c}}$ & $\begin{array}{c}\text { Temp. } \\
\left({ }^{\circ} \mathrm{C}\right)\end{array}$ & $\begin{array}{c}\text { Rotating Speed } \\
(\mathrm{RPM})\end{array}$ \\
\hline Start-Up & $0-30$ & 32 & $32-23.5$ & $0-8.5$ & $0-0.26$ & $35-35.5$ & $1800-2400$ \\
\hline $\begin{array}{l}\text { Steady } \\
\text { Operation }\end{array}$ & $30-60$ & 32 & 23.5 & 8.5 & 0.26 & 35.5 & $2400-2500$ \\
\hline Overload & $60-90$ & 32 & $23.5-28$ & $8.5-4$ & $0.26-0.13$ & $35.5-34.9$ & $2500-4500$ \\
\hline Restoration & $\begin{array}{c}\text { After } \\
90 \mathrm{~s}\end{array}$ & 32 & 23.5 & 8.5 & 0.26 & 35.5 & $2400-2500$ \\
\hline
\end{tabular}

The results show that the control system can work effectively to monitor and control the dehumidifier's working conditions and the applied I/O control methods are able to regulate the operating status of the dehumidifier by sending DAQ control commands and maintaining normal operation. Therefore, this case study verifies that the system has been developed successfully.

\subsection{Mobile control via the cellular phone network}

Figure 10 shows the programme applied at the server-side host computer for transmitting the working condition data to the mobile phone client. The programme is developed based on XML with the LabVIEW's Write Response and the XML Out methods, which allow the data to be transferred online. When the host computer receives a request from a mobile phone, the XML Output method is triggered; then the XML code is compiled in the Write response format and stored in the Write response buffer within the computer memory. Subsequently, the XML code remaining in the buffer is sent to the mobile phone via the Flash Out method.

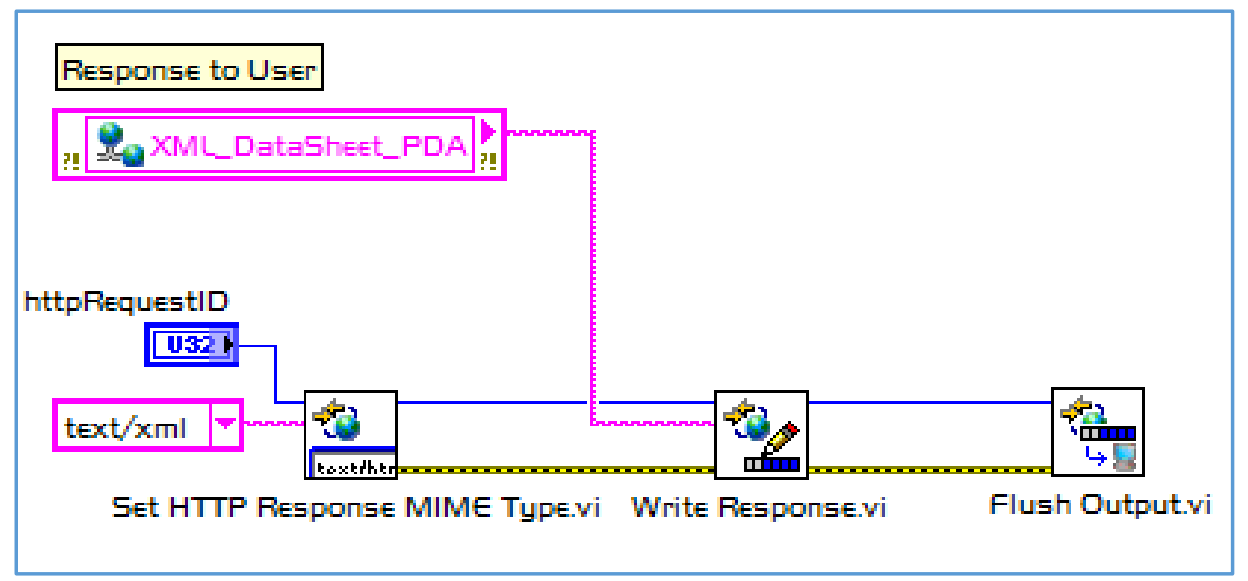

Figure 10: Transmission of working condition data to the mobile phone client with the XML code which is developed using LabVIEW, a programming software based on G language 


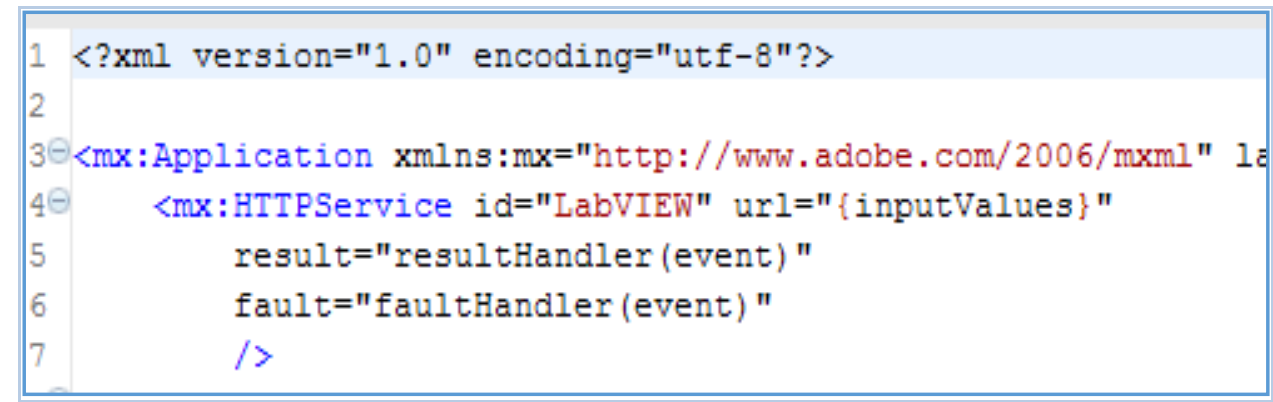

Figure 11: Establishment of the remote connection from the mobile phone to the host computer utilising the REST-based web service

Figure 11 shows the script code for mobile phones to create a connection to the host computer via the REST-based web service, in order to retrieve the working condition data. Within the code, the user interface for the connection with the host computer is specified as the web service of LabVIEW, which is allowed to connect the mobile phone app with MXML language.

As shown in Figure 12, a Nokia phone is utilised to demonstrate the online interactive functions of the system. Although the working conditions of the dehumidifier can be adjusted by the system automatically, the technicians/engineers, under some circumstances, will need to remotely test the system. Furthermore, the user might wish to set different levels of humidity within the room. To meet such requirements, the system allows authorised users to control the dehumidifier with their smartphones.

On the user interface of the mobile phone, there are some parameters and commands for the system control, such as 'Step control', 'Decelerate by $5 \%$ ', 'lower limit of the $\Delta \mathrm{H}^{\prime}$, etc. These parameters and commands allow the user to remotely access the system using the mobile phone. For instance, when 'Step control' was enabled and 'Decelerate by 5\%' was triggered, the fan's speed decreased by $5 \%$ immediately, as shown in Figure 13. When 'Video Stream' was enabled in the mobile phone, the video for monitoring the dehumidifier operation was streamed to the Nokia mobile phone, as shown in Figure 14.

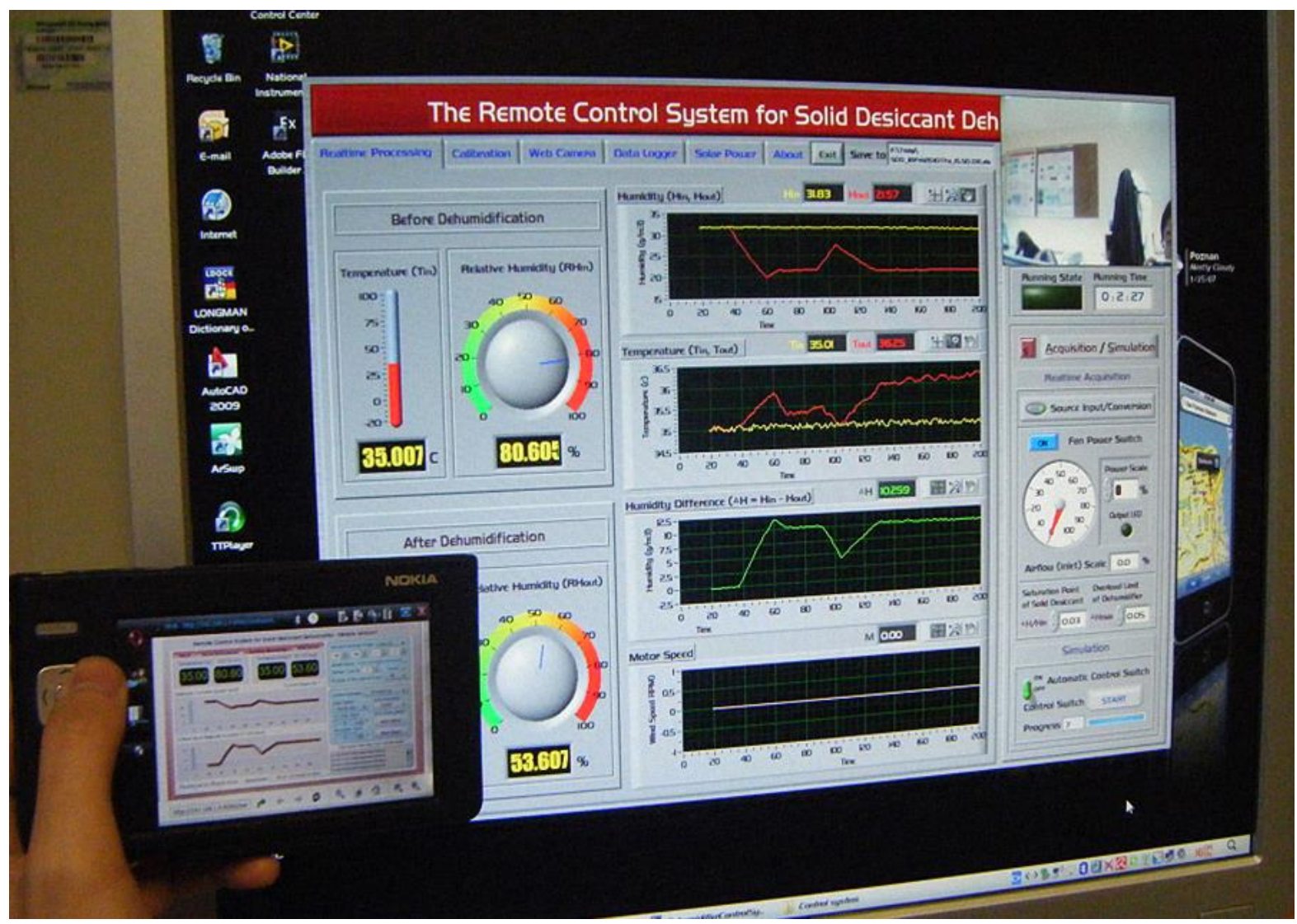

Figure 12: Retrieving working condition data from the host computer via a Nokia phone 


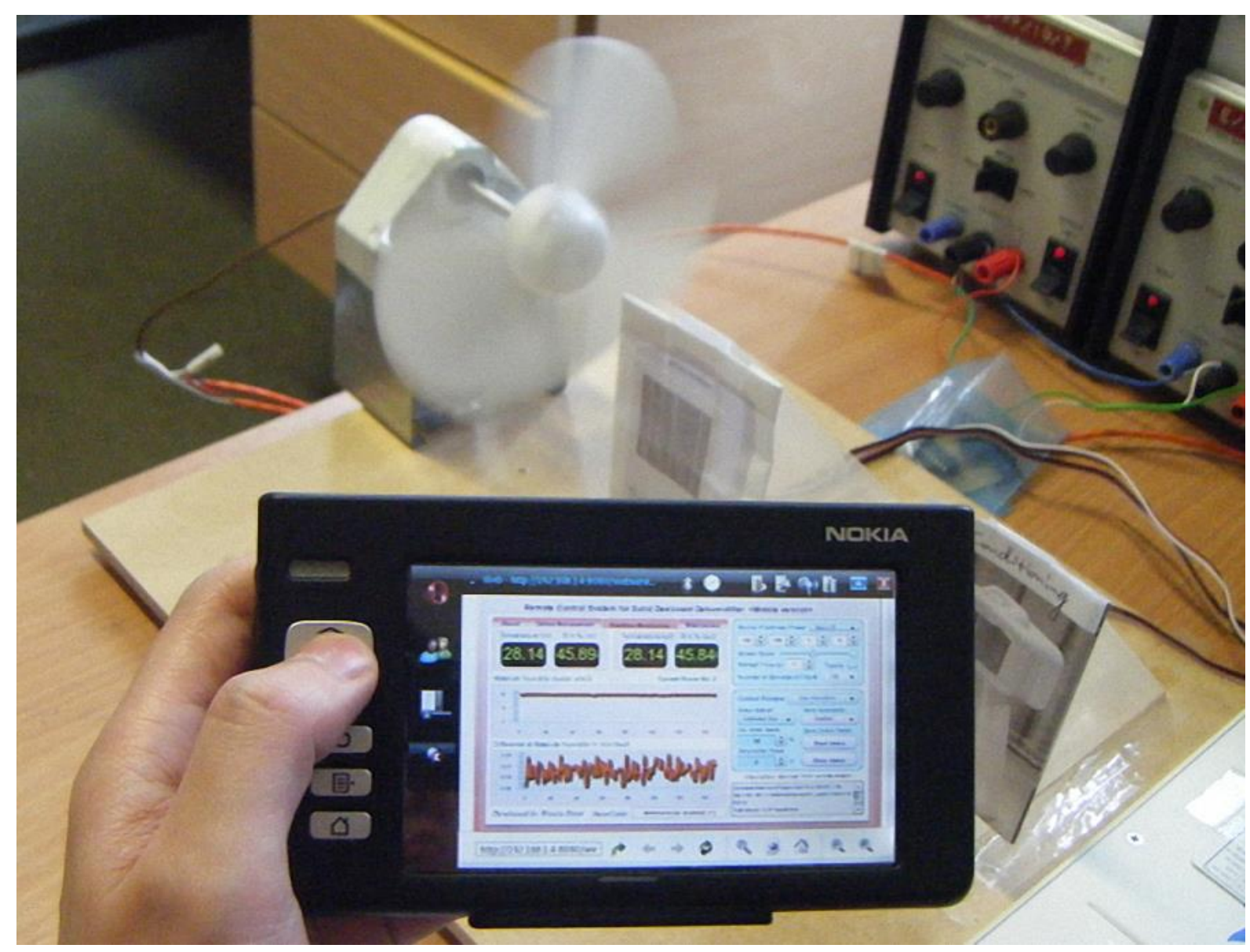

Figure 13: Instant control of fan speed with a Nokia phone

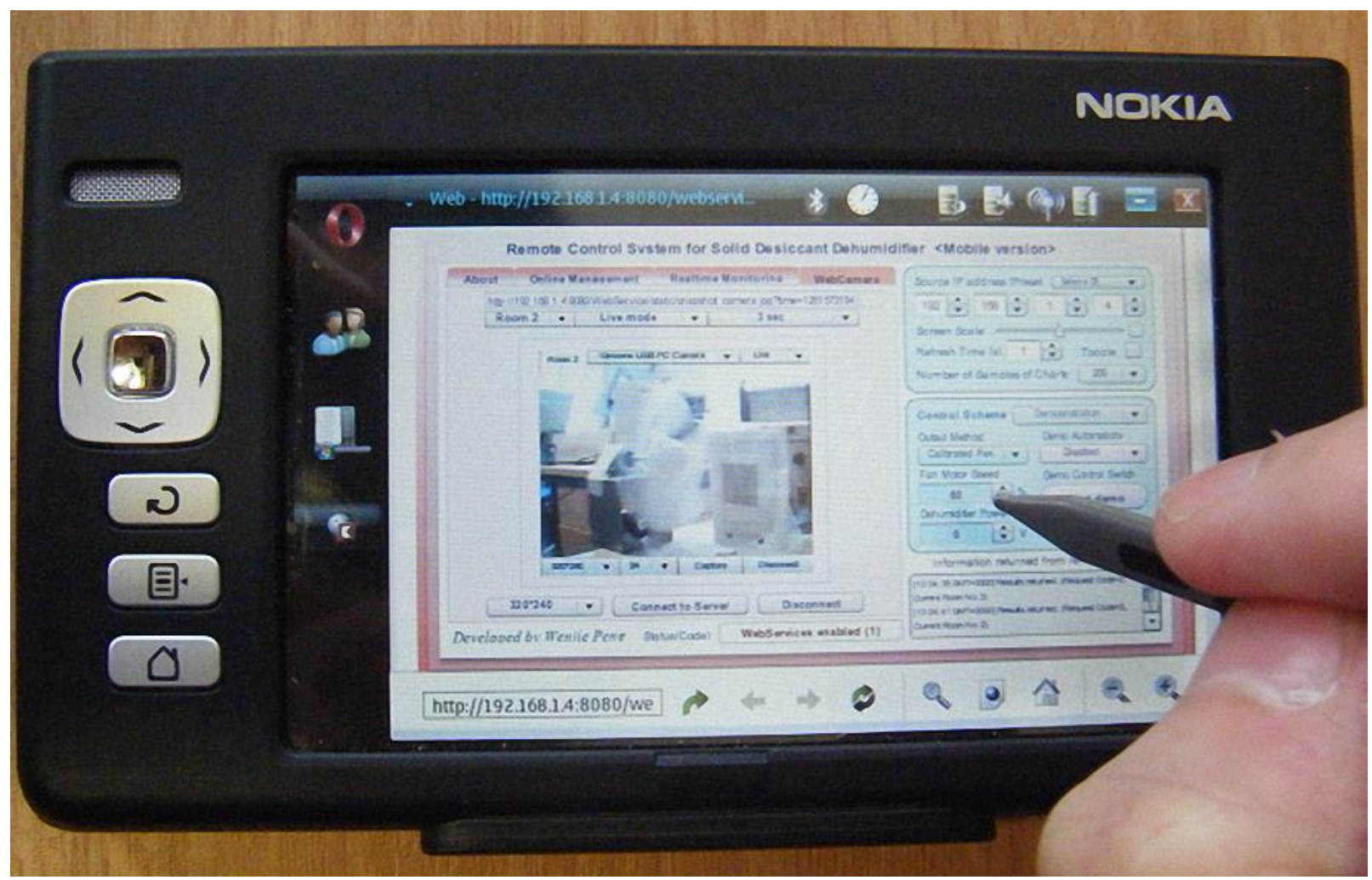

Figure 14: Video stream shown on the Nokia phone for on-site monitoring 


\section{Conclusions}

This paper reports a novel remote monitoring system of a solid desiccant dehumidifier for air-conditioning in low/zero carbon emission buildings, in order to overcome the problems of water evaporative cooling when used to control the temperature and humidity of working / living spaces. The system applies advanced information technologies (IT), such as sensor fusion, digital I/O (input/output) communication and mobile access control methods, to monitor and regulate the internal conditions of air-conditioned buildings with the major benefit of reducing carbon emissions in the buildings. Related software has been successfully developed by the authors. This system has been approved to be used with water-cooled air conditioning systems allowing effective operation of desiccant dehumidification and user friendly control of a building's working / living environment.

This research has made the following contributions to knowledge:

(1) Improvement of the effectiveness of water-cooled air conditioning systems. Currently, the application of water evaporative cooling is limited in the UK and some European countries due to higher air humidity in those areas, which significantly affects the operation of water-cooled air conditioning systems and hence reduces user satisfaction with such systems. To address this issue, an effective solid desiccant dehumidification method is developed, which overcomes the problems of current water evaporative cooling units.

(2) Improvement of water evaporative cooling systems from a user satisfaction perspective. Mobile phone and sensing technologies have not been available for use with water evaporative cooling systems to date, which limits the flexibility of individuals to remotely and efficiently control the building's environment. To overcome this, sensor fusion and mobile phone communication technologies are utilised, which address the problem.

(3) Contribution to the remote monitoring and control methods for regulating working conditions of the system. Although remote control is applied in many fields, this method is not currently available in the application of water-cooled air conditioning and has not been investigated in the existing literature reviews. The relevant IC technologies, such as signal data processing, digital I/O, and onsite visualisation are applied to detect the working status of solid desiccant dehumidification units online, which ensures the ability to remotely control the operation of air-conditioning systems.

The use of water to replace the coolant in traditional air condition can reduce carbon emissions, which is an important advantage of the system reported in this paper. As part of the future work, detailed calculation and comparison will be conducted to show how much carbon emission could be saved and the exact energy efficiency could be achieved with the sensor fusion and mobile communication technology.

Future efforts will focus on the application of solid desiccant dehumidification technology, which is developed in this project, to the development of environmentally friendly technologies for thermal comfort. Due to the small amount of electricity required, it is anticipated that the system can be driven using renewable energy sources, such as solar energy. The relevant technology has not been fully investigated and further research is needed.

The research reported in this paper could be of interest to readers who are investigating methods to overcome the problems of current water evaporative cooling. This research will help readers to understand how mobile phone and remote sensing technologies can be utilised for the control of water-based air conditioning systems.

\section{Acknowledgements}

The authors acknowledge the research grant received from the UK East Midlands' Sustainable Construction iNET programme supported by EU Regional Development Funds (project number: 01-DSG R1806). 


\section{References}

[1] Moo-Yeon Lee, Dong-Yeon Lee, 'Review on Conventional Air Conditioning, Alternative Refrigerants, and $\mathrm{CO}_{2}$ Heat Pumps for Vehicles', Advances in Mechanical Engineering, 2013:1-15. January 2013

[2] Alex Benjamin Wilson, 'Improving energy performance of buildings', EU Legislation in Progress, Fourth edition, EPRS (European Parliamentary Research Service), 19 July 2018

[3] Katja Frieler, 'This is what will happen to the climate in the next 100 years', The Conversation, $11^{\text {th }}$ December 2015, http://theconversation.com/this-is-what-will-happen-to-the-climate-in-the-next-100years-52051 (Accessed 3rd December 2018)

[4] Energy Saving Trust, The Clean Growth Plan: A "2050-ready" new-build homes policy, p.p. 1-4, May 2017

[5] Residential Chillers: Another Cooling Option for Your Home, Perfect Home HVAC Design, http://www.perfect-home-hvac-design.com/chilled-water-air-conditioning.html [Accessed 09 July 2018]

[6] Airwell Water cooled Air Conditioning, P\&M Coppack Air Conditioning Ltd, https://www.pmcoppack.com/sub-category/0000000386/0000000933/air-conditioning/water-cooled [Accessed 09 July 2018]

[7] Chiller Air Conditioning Systems, LG UK Business, https://www.lg.com/uk/business/air-conditioningsystems/chiller [Accessed 09 July 2018]

[8] W. Peng, D. Su, "Internet-based Remote Monitoring System of Thermo-Electric-Generations (TEGs) with Mobile Communication Technology", International Journal of Materials and Product Technology, Inderscience Enterprises Ltd. Vol. 53, No. 2, 2017

[9] D. Su, W. Peng: 'Remote Machine Condition Monitoring Using Wireless Web Technology', Key Engineering Materials, Vols. 419-420 (2015), pp. 745-748

[10] Shuang Hua Yang, Chengwei Daia and Roger P. Knott, 'Remote maintenance of control system performance over the Internet', Control Engineering Practice, Volume 15, Issue 5, May 2010, Pages 533544

[11] J.S. Rao, M. Zubair, C. Rao, 'Condition monitoring of power plants through the Internet', Integrated Manufacturing Systems, Volume: 14, Issue: 6, 2003, Page: 508 - 517

[12] Qiang Miao, Viliam Makis, 'An application of the modulus maxima distribution in machinery condition monitoring', Journal of Quality in Maintenance Engineering, 2005, Volume: 11, Issue: 4, Page: 375 - 387

[13] Sustainable Construction iNET, The Sustainable Construction iNET Higher Education Collaboration Fund confirmation letter for the project entitled 'TBC-Development a novel system of solid desiccant dehumidification for low carbon emission buildings', 10 ${ }^{\text {th }}$ October, 2009

[14] Min-Chun Pan, 'Remote online machine condition monitoring system', Measurement, Volume 41, Issue 8, October 2015, Pages 912-921

[15] O. Amer, R. Boukhanouf, and H. G. Ibrahim, 'A Review of Evaporative Cooling Technologies', International Journal of Environmental Science and Development, Vol. 6, No. 2, February 2015

[16] N. Lechner, Heating, Cooling, Lighting: Sustainable Design Methods for Architects, 3rd ed. New Jersey, U.S.A.: Wiley, 2016, ch. 10, pp. 276-293

[17] UK Visa and Immigration Centre (UVIC), 'Climate Characteristics of Different UK Regions', Life in the UK, Immigration, UVIC (2013-2015). http://uvic.co.uk/life-in-the-uk/climate-characteristics-of-different-ukregions (Accessed 3rd December 2018) 
[18] Europe Humidity: Find Local Weather,

https://www.findlocalweather.com/weather_maps/mapfetch.php?conditions=humidity\&name=europe\&deg rees $=\mathrm{F}$ (Accessed 16th October 2018)

[19] A. Afram and F. Janabi-Sharifi, 'Theory and applications of HVAC control systems - A review of model predictive control (MPC)', Journal of Building and Environment, Volume 72, February 2014, p.p. 343-355

[20] HVAC Technical Institute 2018, '7 Must-Have Apps for Every HVAC Technician', https://www.hvactech.com/7-must-have-apps-for-every-hvac-technician (Accessed 16th October 2018)

[21] I. STEWART, 'Top Mobile Apps for HVAC', MOBILE \& TECH, 18 October 2016

List of Abbreviations

\begin{tabular}{|l|l|}
\hline AI & Analogue Input \\
\hline API & Application Programming Interface \\
\hline CFC & Chlorofluorocarbon \\
\hline DAQ & Data Acquisition \\
\hline DC & digital counter \\
\hline FMS & Flex Media Server \\
\hline GUI & graphic user interfaces \\
\hline HFC & Hydrofluorocarbons \\
\hline HTTP & Hypertext Transfer Protocol \\
\hline HVAC & Heating, Ventilation, and Air Conditioning \\
\hline I/O & Input / Output \\
\hline IT & Information Technology \\
\hline NI & National Instrument \\
\hline REST & Representational State Transfer \\
\hline RH & Relative Humidity \\
\hline RMS & Root Mean Square \\
\hline XML & Extensible Mark-up Language \\
\hline
\end{tabular}

